\title{
Understanding age-related diseases: report of the 2015 Ageing Summit
}

\author{
Lies Lahousse ${ }^{1,2}$, Emmanuel Moyse $e^{3,4}$, Slavica Krantic ${ }^{5}$ and \\ Guy G. Brusselle $1,2,6$
}

Affiliations: 'Dept of Respiratory Medicine, Ghent University Hospital, Ghent, Belgium. ${ }^{2}$ Erasmus Medical Center, Dept of Epidemiology, CA Rotterdam, The Netherlands. ${ }^{3}$ PRC Laboratory, University François Rabelais/Centre INRA of Tours, Nouzilly, France. ${ }^{4}$ Cordeliers Faculty of Medicine, University Paris-Descartes, Paris, France. ${ }^{5}$ Cordeliers Research Centre, INSERM-Paris-Descartes-University, Paris, France. ${ }^{6}$ Dept of Respiratory Medicine, Erasmus Medical Center, CA Rotterdam, The Netherlands.

Correspondence: Lies Lahousse, Ghent University Hospital - Respiratory Medicine, De Pintelaan 185 K12-7 Ghent 9000, Belgium. E-mail: lies.lahoussedugent.be

@ERSpublications

To understand age-related diseases, we must explore how ageing fosters conditions from which these diseases sprout http://ow.ly/UbLYu

The Ageing Summit was held on 10-12th February, 2015 in London. The aim was to discuss advances in the understanding of ageing and the intriguing link with concomitant disorders of the ageing brain, heart and lung. The summit encompassed four thematic sessions of research: cell biology, anti-ageing processes, (epi)genetic regulation and age-related diseases. Based on the nine hallmarks of ageing, this report provides a synopsis of the multidisciplinary meeting on the ageing process, and highlights the most relevant results related to chronic obstructive pulmonary disease (COPD) and idiopathic pulmonary fibrosis (IPF). Since ageing may mechanistically link COPD and its comorbidities, this overview is of interest to clinicians and researchers.

\section{Genomic instability}

The first hallmark of the ageing process is genomic instability [1]. Since the efficiency of DNA repair processes seems to decline with age, increasing research is performed to understand how the resulting genomic instability affects gene expression and protein function in age-related diseases including lung diseases [2]. This progressive decline in DNA repair with age is even more evident in diseases requiring an increased activation of DNA repair pathways, such as idiopathic pulmonary fibrosis (IPF) [3]. Importantly, pulmonary fibrosis has been linked with several progeroid syndromes [4]. Emphysema is not a common feature of progeroid syndromes (table 1). However, atherosclerosis is not only an important aspect in progeric diseases characterised by genomic instability, but also in age-related diseases such as COPD [5].

Anton J.M. Roks (Erasmus Medical Center, Rotterdam, the Netherlands) investigated the role of genomic instability in age-related vascular disease in mice [6]. Increased diastolic blood pressure and carotid intima media thickness appeared to be associated with increased phosphodiesterase (PDE) 1 levels, caused by the senescence of vascular smooth muscle cells and genomic instability. PDE1C levels were increased in lung and aorta, and PDE activity in lungs was higher in a mouse model of human ageing (Ercc1 ${ }^{d /-}$ versus wild-type mice). Diet restriction improved the vasomotor function of mice aorta during vascular ageing caused by genomic instability.

\section{Telomere attrition}

Most cells have a finite number of cell divisions determined by the length of the DNA-protective telomeres at the ends of the chromosomes. Telomerase is an enzyme complex including telomerase reverse transcriptase

Received: May 272015 | Accepted after revision: Aug 122015

Support statement: Financial thanks to the Special Research Fund-Concerted Research Action of Ghent University (BOF/ GOA 01G02714). Lies Lahousse is a Postdoctoral Fellow of the Research Foundation - Flanders (FWO 12E3616N).

Conflict of interest: Disclosures can be found alongside the online version of this article at erj.ersjournals.com 
TABLE 1 Hallmarks of ageing in COPD and idiopathic pulmonary fibrosis (IPF)

\begin{tabular}{lcc} 
Hallmarks of ageing & COPD & IPF \\
\hline Genomic instability & + & ++ \\
Telomere attrition & ++ & +++ \\
Epigenetic alterations & +++ & +++ \\
Loss of proteostasis & ++ & ++ \\
Deregulated nutrient-sensing & + & ++ \\
Mitochondrial dysfunction & ++ & $\mathbf{+}$ \\
Cellular senescence & ++ & ++ \\
Stem cell exhaustion & + & +++ \\
Altered intercellular communication & +++ & +++ \\
\hline
\end{tabular}

COPD: chronic obstructive pulmonary disease. Hallmarks taken from [1]. Hallmarks indicated with +++ are most likely, ++ very likely, + likely and \pm less likely to be involved in the pathogenesis of COPD/IPF. Note 1) both COPD and IPF are heterogeneous pulmonary diseases, implicating that the relative importance of each hallmark of ageing might depend on the underlying phenotype or endotype; and 2) studies directly comparing the relative contribution of specific hallmark(s) of ageing in both COPD and IPF are scarce.

(TERT), which counteracts the telomere shortening occurring at each cellular division. Telomere shortening is accelerated in age-related diseases including cardiovascular disease, diabetes, pulmonary fibrosis and COPD $[4,7]$. In addition, Gabriele Saretzki (Institute for Ageing and Health, Newcastle, UK) examined the protective role of TERT in brain ageing and neurodegeneration [8]. Experimental TERT overexpression in vitro suppressed oxidative stress, mitochondrial DNA damage and apoptosis [9]. In vivo, TERT-deficient mice had increased oxidative stress in the brain and decreased memory upon ageing, which could be reversed by increasing TERT by dietary restriction or treatment with rapamycin. TERT is expressed in neurons and activated microglia of rodent and human brains, but is absent in astrocytes.

Multiple researchers presented work on the association between vascular ageing, chronic inflammation, oxidative stress and telomere shortening. Woodring E. Wright (UT Southwestern Medical Center, Dallas, TX, USA) added to the discussion that telomere shortening can progressively affect gene expression, during a prolonged period of time before telomeres become short enough to generate DNA damage signals. More research is needed to understand how the telomere position or conformation interacts with the expression of genes located ten mega base pairs further into the subtelomeric region.

\section{Epigenetic alterations}

DNA methylation, chromatin remodelling and silencing by non-coding RNAs represent epigenetic changes, which influence gene expression [1]. Associations between epigenetic alterations, ageing and age-related diseases are investigated with growing interest. In lung tissue, correlations were found between age and DNA methylation, and different DNA methylation profiles were observed in patients with IPF and COPD (table 1) [10-12]. Secondly, histone proteins regulate the chromatin structure and thereby the level of gene transcription. Histones generally decline by number with ageing and changes in histone acetylation, methylation or phosphorylation may further account for the looser, more transcriptionally active chromatin structure with ageing [7]. In addition, sirtuins (type III histone deacetylases) have been associated with ageing, COPD, IPF and neurodegenerative diseases [13-15]. Adalberto Merighi (University of Turin, Turin, Italy) found that the phosphorylation of the histone H2AX is an early response to DNA damage and a senescence marker in the mouse brain. However, in senescent subventricular zone neural cells, which had an extremely long cell cycle and slow proliferation rate, the H2AX phosphorylation was primarily related to DNA repair. A better understanding of the different histone modifications in age-related diseases (including the reduced histone deacetylases in COPD) is required before selective inhibitors of histone modification enzymes currently under development, can be used successfully [7].

\section{Loss of proteostasis}

Proteostasis describes the range of processes by which cells control protein (un)folding and degradation. The ability to handle proteostatic stress caused by constant mechanical stress or environmental stressors, such as smoking, declines with age [16]. Kelvin J.A. Davies (University of Southern California, Los Angeles, CA, USA) elaborated on these declining stress responses during ageing. The proteasome efficiency is acutely increased after oxidative stress in young mammalian cells, which makes them temporarily more resistant to oxidative damage. However, in older cells these adaptational responses mediated by the nuclear transcription factor (Nrf) 2 become ineffective. A defective Nrf2 response has been observed in many age-related diseases including cardiac failure, diabetes, chronic renal disease and 
COPD. Moreover, restoring this Nrf2-associated redox imbalance might reverse age-related pulmonary fibrosis [17]. Davies added that oxidative damage starts accumulating only in the last third of life, suggesting that proteasome efficiency does not decrease linearly with age.

\section{Deregulated nutrient sensing}

The somatotrophic axis (growth hormone and insulin-like growth factors (IGF)) and its downstream intracellular effectors including phosphoinositide-3-kinase (PI3K)-protein kinase B (AKT)-mammalian target of rapamycin (mTOR) and FOXO are linked to longevity in humans and model organisms [1]. As insulin and IGF-1 signalling are glucose-sensing, mTOR is amino acid-sensing and the related AMPK and sirtuins are low energy-sensing (high AMP and $\mathrm{NAD}^{+}$levels, respectively), deregulations of these nutrient-sensing systems emphasise the importance of trophic and bioenergetic pathways on longevity [1]. Multiple abstracts on the ageing meeting investigated components or potential inhibitors of this pathway, including caloric restriction.

Caloric restriction was cited to have anti-ageing effects and to be protective against cardiovascular disease, diabetes, neurodegeneration and cancer. Jamie L. Barger (LifeGen Technologies, Madison, WI, USA) observed that many mitochondrial energy metabolism pathways were upregulated and two inflammatory pathways downregulated in flies, rats, and rhesus monkeys under caloric restriction. However, the translation of these life-prolonging effects of caloric restriction in animals towards humans affected by age-related diseases is less clear. For example, the increased work of breathing in COPD patients already requires additional calories to maintain weight. Moreover, vitamin deficiencies (e.g. vitamin D deficiency) are highly prevalent in patients with COPD. Therefore, COPD patients might rather benefit from a healthy diet rich in fruit, vegetables and antioxidants to activate anti-ageing processes (sirtuin 1), than from sole caloric restriction [7].

Isabel Varela-Nieto (Instituto de Investigaciones Biomédicas “Alberto Sols”, Madrid, Spain) discussed the association of decreased IGF-1 with age-related cochlear degeneration and hearing loss, which affects half of the elderly population ( $>65$ years). IGF-1 deficits increase neuroinflammation; in contrast, experiments in mice with an IGF-1-based treatment were successful and open new possibilities for preventing/treating hearing loss in humans [18]. Although IGF-1 deficiency is rare, levels of IGF-1 generally decrease by age in healthy humans [19]. In contrast, enhanced IGF-1 or growth factor signalling has been associated with pulmonary fibrosis in mice and has surprisingly been observed in muscles of patients with COPD [4, 20].

The retina maintains a fine balance between high metabolic activity and damage due to ultraviolet light, oxidative stress, toxins and shear forces. Jeremy M. Sivak (University of Toronto, Toronto, ON, Canada) presented the eye as a sensitive early indicator for many ageing processes. Glaucoma is an age-related, neurodegenerative disease of the retina that impacts ganglion cells. Sirtuin 1 binds and activates peroxisome proliferator-activated receptor $\gamma$ coactivator (PGC)- $1 \alpha$, a master homeostasis regulator that is decreased in the retina of rodent glaucoma models. PGC- $1 \alpha$ knockout mice show increased vulnerability of retinal ganglion cells, whereas PGC- $1 \alpha$ stimulates antioxidant activity of retinal astrocytes [21]. Sirtuin 1 is reduced by oxidative stress and low levels were observed in many age-related diseases including COPD, atherosclerosis, cardiac failure, diabetes, osteoporosis, chronic kidney disease and Alzheimer's disease [7].

\section{Mitochondrial dysfunction}

Irene Maeve Rea (Queens University Belfast, Belfast, UK) presented genes involved in longevity resulting from the European Union-funded Genetics of Healthy Ageing project [22]. She described the key requirements to reach the age of 90: having a good genetic and nutritional profile and avoiding cardiovascular disease, cancer and infectious diseases by maintaining a competent immunological system. Polymorphisms, which account for approximately $25 \%$ of the variation in human lifespan, were discovered in the following specific cellular functions: stress response, hormone biosynthesis, inflammatory and immunological responses, and metabolism and mitochondrial functioning. In addition to being the cell's biggest energy suppliers, mitochondria are very important for the cellular adaptation to environmental challenges [2].

The age-related decline in mitochondria may lead to a loss of mitochondrial metabolic capacity and response to genomic and proteomic stress, thereby contributing to frailty during ageing [2]. Frailty can be defined as the disability to compensate function loss. Frail elderly people have more falls, hospitalisations and an increased risk of mortality [23]. The evaluation of the frailty status is required for optimal management of patients with age-related diseases, including elderly patients with IPF or COPD [24]. Importantly, frailty predicts mortality in subjects with COPD regardless of the severity of airflow limitation or presence of comorbidities [25].

In addition to the brain, endocrine and immune system, skeletal muscle is also involved in the development of frailty [26]. George Kuchel (University of Connecticut, Storrs, CT, USA) shared observations that muscle performance typically declines more than muscle mass in the elderly. Mitochondrial dysfunction in the muscle results indeed in an increased release of reactive oxygen species and oxidative damage, which is thought to play a key role in ageing [27]. Michael Singer (Queen's University Kingston, Kingston, ON, 
Canada) further underlined the importance of the frailty concept by stating that there are no specific longevity genes, but that ageing is a matter of your ability to respond to environmental cues. This ability, or lack of ability as in frailty, may be determined more by the epigenome than by the genome.

\section{Cellular senescence}

Ken Parkinson (Queen Mary University of London, London, UK) elaborated on the cellular senescence hallmark of ageing by comparing the metabolomes of senescent fibroblast cells with those of quiescent and proliferating cells $[1,28]$. Cellular senescence is defined as a permanent cell cycle arrest through tumour suppressor pathways and is characterised by an altered metabolism and a senescence-associated secretory phenotype (SASP), which influences surrounding cells. Cellular senescence can mediate beneficial or detrimental effects depending on the contextual roles and cell type [2]. For example in IPF pathogenesis, cell senescence is accelerated in alveolar epithelial cells leading to detrimental re-epithelialisation [15]. The SASP of senescent cells is believed to contribute to inflammageing and accelerated ageing due to its pro-inflammatory properties [7]. Furthermore, the SASP response is associated with an activation of the nuclear factor $\kappa \mathrm{B}$ pathway, which is involved in many age-related diseases including COPD and cardiovascular disease [7].

\section{Stem cell exhaustion}

The concepts of cellular senescence and stem cell exhaustion were combined by Richard Richardson (McGill University, Montreal, QC, Canada) in his talk on the predominance of accelerated ageing in highly proliferative tissues. Ageing is characterised by reduced stem cell proliferation and increased cell death to avoid carcinogenesis in damaged tissue [29]. The progenitor cell demand-to-supply ratios might even be a risk indicator of cancer and potentially explains the bimodal age-incidence curves of cancers such as leukaemia. However, the replicative senescence of stem cells comes at cost of loss of organ mass. Organs with shorter cell turnover exhibit greater replicative senescence and subsequent greater age-related mass loss [29]. Bone-marrow derived endothelial progenitor cells are reduced in both COPD and IPF patients, and the observation that diseases of accelerated aging are characterised by an excessive loss of bone, muscle or lung is food for thought [4].

\section{Altered intercellular communication}

Ageing is accompanied by dysregulations in neuro-hormonal signalling, immune surveillance and extracellular matrix turnover [1]. Cheryl Frye (University of Alaska, Alaska, AK, USA) focussed on the role of neurosteroids in the ageing brain by stating that the brain is an endocrine organ that can generate de novo steroids in response to environmental and social challenges to regulate homeostasis. Furthermore, ageing is associated with a reduced capacity of the brain to produce these neurosteroids. Increasing evidence shows that sex steroids including progesterone and its brain-derived metabolites, indeed influence cognitive functions in murine models.

Jennifer Pocock (UCL Institute of Neurology, London, UK) focussed on immunesurveillance by investigating the role of microglia, the macrophages of the brain and the communicators between the immune system and the brain. Dysfunctional, dystrophic and senescent microglia with low turnover characterise brain ageing. The main phenotype of microglia, which represents $16 \%$ of the total cells in the human adult central nervous system, changes from a M2 (protective) to a M1 (pro-inflammatory) phenotype during ageing. Pro-inflammatory microglia release cytokines such as interleukin- 6 and tumour necrosis factor- $\alpha$ and growth factors such as transforming growth factor- $\beta$. Age-related diseases, including COPD and IPF, are also marked by the activation of such pro-inflammatory mediators (table 1) $[4,15]$.

Finally, the implication of protease-mediated extracellular matrix (ECM) degradation in age-related, chronic inflammatory disorders was presented by David Granville (University of British Columbia, Vancouver, BC, Canada). They observed that granzyme B contributes directly and indirectly to ECM degradation in accelerated skin ageing upon ultraviolet light irradiation. Granzyme B knockout mice were protected against the formation of wrinkles and the loss of dermal collagen density [30]. The accumulation of matrix proteins is also important in other age-related diseases including arterial ageing, discussed by Majd AlGhatrif (National Institute on Ageing, Bethesda, MD, USA), where it leads to fibrosis of the intimal walls and thereby increased arterial stiffness [31].

ECM degradation leads also to a progressive loss of the elastic fibres in the senile lung. A poster of Masataka Sugimoto (National Centre for Geriatrics and Gerontology, Obu City, Japan) presented a senile lung mouse model, in which they were able to visualise senescent cells and induce their elimination from the lung tissue at a certain point (using a luciferase and diphtheria toxin receptor cell-knockout system under the control of CDKN2A). After elimination of senescent cells from lung tissue of old animals, elastic fibres were recovered and the alveolar size was reduced to near those of young mice. Since ECM dysregulation is a key feature of 
both IPF and COPD, improving our understanding of the onset, maintenance and consequences of an imbalanced ECM are crucial for developing potential new therapies [4].

In conclusion, to become healthy and happy centenarians, we need to postpone the onset of age-related diseases. Our weapons are given at birth, though the attrition rate is largely determined by the environmental factors we encounter and the fitness by which we battle them. Ageing increases the vulnerability to disease but is not a disease itself. Frailty reflects better the state of vulnerability in late life than chronological age. Meetings such as the Ageing Summit keep us updated on the mechanisms of ageing, increase our understanding of age-related diseases and provide opportunities for therapeutic interventions which target multiple chronic age-related diseases with shared pathogenetic mechanisms.

\section{References}

López-Otin C, Blasco MA, Partridge L, et al. The hallmarks of aging. Cell 2013; 153: 1194-1217.

2 Thannickal VJ, Murthy M, Balch WE, et al. Blue journal conference. Aging and susceptibility to lung disease. Am J Respir Crit Care Med 2015; 191: 261-269.

Thannickal VJ. Mechanistic links between aging and lung fibrosis. Biogerontology 2013; 14: 609-615.

Meiners S, Eickelberg O, Königshoff M. Hallmarks of the ageing lung. Eur Respir J 2015; 45: 807-827.

Lahousse L, van den Bouwhuijsen QJ, Loth DW, et al. Chronic obstructive pulmonary disease and lipid core carotid artery plaques in the elderly: the Rotterdam Study. Am J Respir Crit Care Med 2013; 187: 58-64.

6 Durik M, Kavousi M, van der Pluijm I, et al. Nucleotide excision DNA repair is associated with age-related vascular dysfunction. Circulation 2012; 126: 468-478.

7 Barnes PJ. Mechanisms of development of multimorbidity in the elderly. Eur Respir J 2015; 45: 790-806.

8 Spilsbury A, Miwa S, Attems J, et al. The role of telomerase protein TERT in Alzheimer's disease and in tau-related pathology in vitro. J Neurosci 2015; 35: 1659-1674.

9 Saretzki G. Extra-telomeric functions of human telomerase: cancer, mitochondria and oxidative stress. Curr Pharm Des 2014; 20: 6386-6403.

10 Sanders YY, Ambalavanan N, Halloran B, et al. Altered DNA methylation profile in idiopathic pulmonary fibrosis. Am J Respir Crit Care Med 2012; 186: 525-535.

11 Qiu W, Baccarelli A, Carey VJ, et al. Variable DNA methylation is associated with chronic obstructive pulmonary disease and lung function. Am J Respir Crit Care Med 2012; 185: 373-381.

12 Christensen BC, Houseman EA, Marsit CJ, et al. Aging and environmental exposures alter tissue-specific DNA methylation dependent upon CpG island context. PLoS Genet 2009; 5: e1000602.

13 Rajendrasozhan S, Yang SR, Kinnula VL, et al. SIRT1, an antiinflammatory and antiaging protein, is decreased in lungs of patients with chronic obstructive pulmonary disease. Am J Respir Crit Care Med 2008; 177: 861-870.

14 Guarente L. Sirtuins, aging, and medicine. N Engl J Med 2011; 364: 2235-2244.

15 Minagawa S, Araya J, Numata T, et al. Accelerated epithelial cell senescence in IPF and the inhibitory role of SIRT6 in TGF- $\beta$-induced senescence of human bronchial epithelial cells. Am J Physiol Lung Cell Mol Physiol 2011; 300: L391-L401.

16 Balch WE, Sznajder JI, Budinger S, et al. Malfolded protein structure and proteostasis in lung diseases. Am J Respir Crit Care Med 2014; 189: 96-103.

17 Hecker L, Logsdon NJ, Kurundkar D, et al. Reversal of persistent fibrosis in aging by targeting Nox4-Nrf2 redox imbalance. Sci Transl Med 2014; 6: 231 ra247.

18 Varela-Nieto I, Murillo-Cuesta S, Rodriguez-de la Rosa L, et al. IGF-I deficiency and hearing loss: molecular clues and clinical implications. Pediatr Endocrinol Rev 2013; 10: 460-472.

19 O'Connor KG, Tobin JD, Harman SM, et al. Serum levels of insulin-like growth factor-I are related to age and not to body composition in healthy women and men. J Gerontol A Biol Sci Med Sci 1998; 53: M176-M182.

20 Doucet M, Russell AP, Leger B, et al. Muscle atrophy and hypertrophy signaling in patients with chronic obstructive pulmonary disease. Am J Respir Crit Care Med 2007; 176: 261-269.

21 Guo X, Dason ES, Zanon-Moreno V, et al. PGC-1alpha signaling coordinates susceptibility to metabolic and oxidative injury in the inner retina. Am J Pathol 2014; 184: 1017-1029.

22 Deelen J, Beekman M, Uh HW, et al. Genome-wide association meta-analysis of human longevity identifies a novel locus conferring survival beyond 90 years of age. Hum Mol Genet 2014; 23: 4420-4432.

23 Lahousse L, Maes B, Ziere G, et al. Adverse outcomes of frailty in the elderly: the Rotterdam Study. Eur J Epidemiol 2014; 29: 419-427.

24 Meyer KC, Danoff SK, Lancaster LH, et al. Management of idiopathic pulmonary fibrosis in the elderly patient: addressing key questions. Chest 2015; 148: 242-252.

25 Lahousse L, Ziere G, Verlinden VJA, et al. Risk of frailty in elderly with COPD: a population-based study. J Gerontol A Biol Sci Med Sci 2015; in press [DOI: 10.1093/gerona/glv154].

26 Clegg A, Young J, Iliffe S, et al. Frailty in elderly people. Lancet 2013; 381: 752-762.

27 MacNee W, Rabinovich RA, Choudhury G. Ageing and the border between health and disease. Eur Respir J 2014; 44: 1332-1352.

28 James EL, Michalek RD, Pitiyage GN, et al. Senescent human fibroblasts show increased glycolysis and redox homeostasis with extracellular metabolomes that overlap with those of irreparable DNA damage, aging, and disease. J Proteome Res 2015; 14: 1854-1871.

29 Richardson RB, Allan DS, Le Y. Greater organ involution in highly proliferative tissues associated with the early onset and acceleration of ageing in humans. Exp Gerontol 2014; 55: 80-91.

30 Parkinson LG, Toro A, Zhao H, et al. Granzyme B mediates both direct and indirect cleavage of extracellular matrix in skin after chronic low-dose ultraviolet light irradiation. Aging Cell 2015; 14: 67-77.

31 Scuteri A, Morrell $\mathrm{CH}$, Orru $\mathrm{M}$, et al. Longitudinal perspective on the conundrum of central arterial stiffness, blood pressure, and aging. Hypertension 2014; 64: 1219-1227. 